\title{
Crecimiento y desarrollo de hijos de adolescentes de clase media-baja. II Parte: 30 meses de vida
}

\author{
Dra. Raquel Burrows A. 1; 'T.M. Laura Leiva B. ; Sr. Ruperto Bravo B. ${ }^{1}$; \\ Dra. Laura Macho F. ${ }^{2}$; Sr. A braham Zvaighaft F. ${ }_{\text {; }}$ Dr. Santiago Muzzo B. ${ }^{3}$
}

Growth and development in children from teenage's mothers

A longitudinal study of children from tecriger motlers bclonging to a middle-low socioeconomic level in their first 30 months of life, was done. Weight, height, height velocity, bone age, plasmatic levels of zinc, cooper and hemoglobin, food intake, sociocconomic lcvel, and stature of parents werc studied. There were not differences in nutritional status, caloric intake, bone age, plasmatic lovels of zinc, cooper and hemoglobin, and stature of parents betwen children of teenage and adult mothers. A progressive decrement in stature and in growth velocity of children of teenage compared with children of adult mothers was observed. A lower socioeconomic level and protein intake was found in children of adolescent mothess that may be playing a role at least in part in the growth failure presented by this children. Etiology cal lactors of this growth failure discussed.

(Key words: Children from teenager mothers. Growth and development).

Se ha descrito en hijos de madres adolescentes un deterioro del crecimiento y desarrollo en la vida pre y post natal temprana ${ }^{1-5}$, al compararlos con hijos de mujeres adultas. Esta diferencia de estatura se acentúa en la medida que la adolescente aumenta el número de hijos y deteriora su nivel socioeconómico ${ }^{11}$. Se ha demostrado, que un control adecuado del embarazo pre$\operatorname{coz}$ en un centro de adolescencia, previene un bajo peso del recién nacido y disminuye las complicaciones obstétricas descritas en estos embarazos ${ }^{12-14}$ Hemos encontrado un crecimiento y desarrollo similar durante el primer año de vida entre hijos de mujeres adolescentes y adultas de nivel socioeconómico medio-bajo. El allegamiento de la adolescente al hogar paterno y el adecuado control de su hijo en el Servicio de Salud, durante este períodó crítico de la vida juegan un rol preventivo importante ${ }^{15}$. Sin embargo, el crecimiento en los hijos de adolescentes comienza a deteriorarse después de los 12 meses de edad, encontrándose una menor velocidad de crecimiento estatural sin deterioro del peso para la estatura, al compararlos con los hijos de adultas ${ }^{16}$. Se sabe que factores genéticos y am. bientales influyen en el crecimiento estatural de un sujeto. Dentro de los factores ambientales la

1. Unidad de Endocrinología, Instituto de Nutrición y Tecnología de los Alimentos, Universidad de Chile.

2. Servicio de Radiología, Hospital Luis Calvo Mackenna. nutrición juega un rol importante, describiéndose que no sólo el déficit calórico puede comprometer el crecimiento de un niño ${ }^{18-20}$ sino también déficit de nutrientes, específicos 21,27 . Nos interesó investigar si el déficit de crecimiento que presentan los hijos de adolescentes se acentúa y estudiar algunos posibles factores etiológicos. Para ello efectuamos un corte transversal a los 30 meses de vida de nuestro seguimiento longitudi. nal de hijos de adolescentes de clase media-baja.

\section{MATERIAL Y METODOS}

Se saleccionó en un corte transversal a los 30 meses de edad 29 hijos de mujeres adolescentes y 27 de adultas, en quienes se evaluó semestralmente desde el nacimiento, su crecimiento $y$ desarrollo 16 . Las madres adolescentes controlaron su embarazo en un Centro de Adolescencia y las adultas en policlínjcos de obstetricia del Servicio de Salud 15. En ambos grupos de preescola. res, se evaluaron el estado nutritivo (P/T), la estatura (T/E) y el ritmo de crecimiento estatural (RC) $y$ se expresaron como porcentajes de adecuación al percentil 50 de las normas OMS para edad y sexo ${ }^{8}$. El nivel Socioeconómico (NSE) se evaluó según la escala de Graffar Modificada29. Se extrajo a cada niño (previo consentimiento de los padres) $3 \mathrm{ml}$ de sangre venosa periférica en ayunas, para la determinación de niveles plasmáticos de Zinc y Cobre por espectrofotometría de absorción atónica y de Hemaglobina por el método de hemoglo Wiener. La maduración ósea se evaluó con una radiografía de carpo izqujerdo de acuerdo al atlas de Greulich y Pyle ${ }^{30}$. Se determinó la ingesta de nutrientes por una encuesta de recordatorio de 24 horas, efectuadas a las madres de estos preescolares. Se midió la estatura de ambos padres y se investigó el origen étnico por los dos apellidos de cada progenitor. El análisis 
estadístico de los resultados se realizó a través de la t de student, $X^{2}$ y prueba de Mann-Whitney.

\section{RESULTADOS}

En la Tabla 1 se observa el NSE de ambos grupos de madres en el momento del parto (año 1981) y 30 meses después (1984). En el año 1981, el NSE de las madres adolescentes y adultas era similar; sin embargo, en la evaluación realizada el año 1984 se detectó que las adolescentes presentan un NSE significativamente más bajo que el de las adultas. Por otro lado, el NSE actual de las madres adolescentes es significativamente menor al que tenian 30 meses atrás $(p<0,02)$.

Con respecto al estado conyugal de las madres (Tabla 2), no hubo diferencias significativas entre adolescentes y adultas, existiendo en la actuali. dad mujeres sin pareja en 20,6 y $14,8 \%$ respectivamente. Un $93,7 \%$ de los hijos de adolescentes $y$ el $100 \%$ de los hijos de adultas viven con sus madres, no existiendo diferencias entre ambos grupos. El estado de la nutrición, analizado por el peso/talla a los 30 meses de vida (Tabla 3), fue similar en hijos de adolescentes y adultas. Si bien, se observó en ambos grupos deterioro del peso para la estatura entre los 3 y los 12 meses, se ha mantenido sin variación durante el último año.

Tabla 2.

Estado actual de las madres

\begin{tabular}{|c|c|c|c|c|}
\hline & \multicolumn{2}{|c|}{ Adolescentes } & \multicolumn{2}{|c|}{ Adultas } \\
\hline & $N$ & $\%$ & $\mathrm{~N}$ & $\%$ \\
\hline - Casada & 18 & 62,2 & 23 & 85,2 \\
\hline Convive & 5 & 17,2 & 0 & 0,0 \\
\hline Separada & 1 & 3,4 & 2 & 7,4 \\
\hline Soltera & 5 & 17,2 & 2 & 7,4 \\
\hline & 29 & 100,0 & 27 & 100,0 \\
\hline & & $\begin{array}{l}\mathrm{gl} \\
\mathrm{p}\end{array}$ & & \\
\hline
\end{tabular}

Tabla 1.

Nivel socioeconómico según Graffar modificado de las madres

\begin{tabular}{|c|c|c|c|c|c|c|}
\hline & \multirow{2}{*}{$\begin{array}{l}\text { Nivel de } \\
\text { Graffar }\end{array}$} & \multicolumn{2}{|c|}{ Adolescentes } & \multicolumn{2}{|c|}{ Adultas } & \multirow[t]{2}{*}{$\mathrm{P}^{*}$} \\
\hline & & $\mathrm{N}$ & $\%$ & $\mathrm{~N}$ & $\%$ & \\
\hline \multirow[t]{3}{*}{ Año 1981 } & $3-4$ & 23 & 79,3 & 21 & 77,8 & \multirow[t]{2}{*}{ N.S. } \\
\hline & $5-6$ & 6 & 20,7 & 6 & 2,2 & \\
\hline & Sub-Total & 29 & 100,0 & 27 & 100,0 & \\
\hline \multirow[t]{2}{*}{ Año 1984} & $3-4$ & 16 & 55,2 & 21 & 77,8 & \multirow{2}{*}{$<0,05$} \\
\hline & $5-6$ & 13 & 44,8 & 6 & 22,2 & \\
\hline & Sub-Total & 29 & 100,0 & 27 & 100,0 & \\
\hline
\end{tabular}

Significación según $\mathrm{X}^{2}$ entre cada grupo en los años 1981 y 1984 .

Significación según test U (Mann-Whitney)

$\mathrm{p}<0.02$ en las adolescentes entre 1981 y 1984.

p N.S. en las adultas entre 1981 y 1984.

Tabla 3.

Porcentaje de adecuación del peso/talla en los hijos de adolescentes y adultas

\begin{tabular}{|c|c|c|c|c|c|}
\hline & \multicolumn{5}{|c|}{ EDAD (meses) } \\
\hline & RN & 3 & 12 & 18 & 30 \\
\hline . Hijos de Adolescentes & $\begin{array}{c}95,3 \pm 6,4 \\
(29)\end{array}$ & $\begin{array}{c}108,6 \pm 11,9 \\
(29)\end{array}$ & $\begin{array}{c}101,6 \pm 9,7 \\
(28)\end{array}$ & $\begin{array}{c}100,2 \pm 7,7 \\
(27)\end{array}$ & $\begin{array}{c}101,5 \pm 7,0 \\
(29)\end{array}$ \\
\hline Hijos de Adultas & $\begin{array}{c}95,9 \pm 8,1 \\
(27)\end{array}$ & $\begin{array}{c}110,1 \pm 11,4 \\
(23)\end{array}$ & $\begin{array}{c}103,7 \pm 8,8 \\
(27)\end{array}$ & $\begin{array}{c}102,9 \pm 7,8 \\
(26)\end{array}$ & $\begin{array}{c}104,2 \pm 9,5 \\
\text { (27) }\end{array}$ \\
\hline $\begin{array}{l}\text { Significación entre perío } \\
\text { Significación entre per io } \\
\text { Significación entte perio }\end{array}$ & $\begin{array}{l}\mathrm{RN} \text { y } 12 \mathrm{M} \\
3 \mathrm{M} \text { y } 12 \mathrm{Mp} \\
3 \mathrm{M} \text { y } 12 \mathrm{M} \mathrm{P}\end{array}$ & $\begin{array}{l}\text { cada grupo de } \\
\text { hijos de adoles } \\
\text { hijos de adulta }\end{array}$ & os & $\begin{array}{l}05 \\
05 \\
25\end{array}$ & \\
\hline
\end{tabular}


El promedio estatural a los 30 meses de edad (Tabla 4), fue significativamente menor en los hijos de adolescentes (tanto varones como mujeres). El ritmo de crecimiento tumbién fue menor en hijos de adulescentes durante los 30 meses de vida y en el último año, al compararlos con los hijos de adultas. La ingesta de nutrientes (Tabla 5) determinada por una encuesta de recordatorio, no reveló diferencias entre hijos de adolescentes y adultas en el promedio de ingesta diaria de calorias, proteínas totales, lípidos e hidratos de carbono. Sin embargo, existe un significativo mayor porcentaje de niños con ingesta de proteínas inferior a $40 \mathrm{~g}$ entre los hijos de adolescentes que en los de adultas ( $p<0,0125)$ (Tabla 6). La ingesta diaria de proteínas totales $y$ animales en los niños con velocjdad de crecimiento estatural igual o inferior al $97 \%$, fue significativamente menor en los hijos de adolescentes que en los de adultas $(\mathrm{p}<0,025)$. (Tabla 7). No hubo diferencias significativas en las concentraciones plasmáticas de Zinc, Cobre.

Tabla 4.

Crecimiento en hijos de adolescentes $y$ adultas a los 30 meses de edad

\begin{tabular}{|c|c|c|c|c|c|}
\hline & \multicolumn{2}{|c|}{ Hijos adolescentes } & \multicolumn{2}{|c|}{ Hijos Adulta } & \multirow{2}{*}{$p(b)$} \\
\hline & Hombre & Mujer & Hombre & Mujer & \\
\hline Talla $(\mathrm{cm})$ & $\begin{array}{c}87,8 \pm 3,6^{a} \\
(14)\end{array}$ & $\begin{array}{c}88,3 \pm 1.7 \\
(15)\end{array}$ & $\begin{array}{l}90,5 \pm 2,1 \\
(12)\end{array}$ & $\begin{array}{c}89,6 \pm 2,1 \\
(15)\end{array}$ & $\begin{array}{l}* 0,025 \\
\underline{\underline{x}} 0,05\end{array}$ \\
\hline . Taila/edad $(\%)$ & \multicolumn{2}{|c|}{$\begin{array}{c}97,0 \pm 3,1 \\
(29)\end{array}$} & \multicolumn{2}{|c|}{$\begin{array}{c}99,3 \pm 2,3 \\
(27)\end{array}$} & $* \overline{\underline{4}} 0,0025$ \\
\hline . Ritmo de crecimiento & $\begin{array}{c}38,6 \pm 3,2 \\
(14)\end{array}$ & $\begin{array}{c}39,0 \pm 2,1 \\
(15)\end{array}$ & $\begin{array}{c}40,8 \pm 2,8 \\
\text { (12) }\end{array}$ & $\begin{array}{c}40,8 \pm 2,7 \\
(15)\end{array}$ & $\begin{array}{l}* 0,05 \\
\pm 0,05\end{array}$ \\
\hline $\begin{array}{l}\text { Ritmo de crecimiento ( } \% \text { ) } \\
\text { en los } 30 \text { meses }\end{array}$ & \multicolumn{2}{|c|}{$\begin{array}{l}95,8 \pm 6,7 \\
(29)\end{array}$} & \multicolumn{2}{|c|}{$\begin{array}{c}100,7 \pm 6,6 \\
(27)\end{array}$} & $* * 0,005$ \\
\hline $\begin{array}{l}\text { Ritmo de crecimjento (\%) } \\
\text { último año }\end{array}$ & \multicolumn{2}{|c|}{$\begin{array}{c}97,0 \pm 15,1 \\
(27)\end{array}$} & \multicolumn{2}{|c|}{$\begin{array}{l}107,6 \pm 16,2 \\
\{26)\end{array}$} & $* \pm 0,01$ \\
\hline
\end{tabular}

Tabla 5.

Ingesta de nutrientes por encuesta de recordatorio

\begin{tabular}{|c|c|c|c|}
\hline & Hijos Adolescentes & Hijos Adultas & $\mathrm{P}(\mathrm{b})$ \\
\hline Proteínas Totales (G) & $\begin{array}{l}44,5 \pm 14,4^{a} \\
\text { (25) }\end{array}$ & $\begin{array}{l}47,8 \pm 10,8 \\
(25)\end{array}$ & N.S. \\
\hline . Calorías (G) & $\begin{array}{c}1.222,5 \pm 288,2 \\
(25)\end{array}$ & $\begin{array}{c}1.224,0 \pm 261,0 \\
(25)\end{array}$ & N.S \\
\hline Hidratos de Carbono (G) & $\begin{array}{c}194,0 \pm \quad 60,1 \\
(25)\end{array}$ & $\begin{array}{c}201,2 \pm \$ 3,1 \\
(25)\end{array}$ & N.S. \\
\hline Lipidos (G) & $\begin{array}{r}38,3 \pm 10,8 \\
(25)\end{array}$ & $\begin{array}{c}36,2 \pm \\
(25)\end{array}$ & N.S. \\
\hline . Proteínas Animales (G) & $\begin{array}{r}25,7 \pm \quad 9,3 \\
(25)\end{array}$ & $\begin{array}{l}28,8 \pm 10,7 \\
\text { (25) }\end{array}$ & N.S. \\
\hline
\end{tabular}

\footnotetext{
(a) $\overline{\mathrm{X}} \pm$ D.E. (Número de Casos)

(b) Significación según $t$ de Student

N.S. No significativo
} 
Tabla 6.

Ingesta proteica por encuesta recordatorio en los hijos a los 30 meses de edad

\begin{tabular}{|c|c|c|c|c|}
\hline \multirow[b]{2}{*}{ Proteínas Totales } & \multicolumn{2}{|c|}{ Hijos Adolestentes } & \multicolumn{2}{|c|}{ Hīos Adultas } \\
\hline & $s ?$ & $\%$ & $\mathbf{N}$ & $\%$ \\
\hline \multirow{4}{*}{$\begin{array}{l}<40 G \\
\geqslant 40 G\end{array}$} & 14 & 51,9 & 5 & 20,0 \\
\hline & 13 & 48,1 & 20 & 80,0 \\
\hline & 27 & 100,0 & 25 & 100,0 \\
\hline & $x^{2}: s$ & g. 1 & $\mathrm{p}$ & 125 \\
\hline
\end{tabular}

hemoglobina y en la maduración ósea entre hijos de adolescentes y adultas (Tabla 8). La talla promedio de los padres fue similar en ambos grupos de nifios (Tabla 9). No hubo diferencias en el origen étnico de los apellidos de los padres, siendo el porcentaje de apellidos amerindios inferior a $5 \%$. Ел los hijos de adolescentes que erecieron con una velocidad inferior o igual al
97\% existió un mayor porcentaje de niños con nivel socioeconómico bajo y miseria, al compararlos con los que lo hicieron a mayor velocidad durante el último año. (Tabla 10).

Tabla 10.

Nivel socjoeconómico (Graffar modificado) en hijos de adolescentes según porcentaje de adecuación del ritmo de crecinjento

\begin{tabular}{|c|c|c|c|c|}
\hline \multirow{3}{*}{ Nivel Graffar } & \multicolumn{4}{|c|}{ Ritmo de ascenso estatural } \\
\hline & \multicolumn{2}{|c|}{$\leqslant 97 \%$} & \multicolumn{2}{|c|}{$<97 \%$} \\
\hline & $\mathbf{N}$ & $\%$ & $\mathrm{~N}$ & $\%$ \\
\hline \multirow[t]{2}{*}{$\begin{array}{l}3-4 \\
5-6\end{array}$} & $\begin{array}{l}6 \\
9\end{array}$ & $\begin{array}{l}40,0 \\
60,0\end{array}$ & $\begin{array}{l}9 \\
3\end{array}$ & $\begin{array}{l}75,0 \\
25,0\end{array}$ \\
\hline & 15 & 100,0 & 12 & 100,0 \\
\hline \multicolumn{5}{|c|}{$\begin{array}{l}\mathrm{X}^{2}=3.86 \\
\mathrm{~g}=\mathrm{l} \\
\mathrm{p}<0.05\end{array}$} \\
\hline
\end{tabular}

Tabla 7.

Ingesta proteica ( $\mathrm{x}$ día) en hijos de adolescentes $y$ adultas con adecuación de incremento estatural $\leqslant 97 \%$

\begin{tabular}{|c|c|c|c|}
\hline & Hijos adolescentes & Hijos de adultas & $\mathrm{Pb}$ \\
\hline Proteínas Totales & $36,6 \pm 12,1 \quad(14)^{a}$ & $46,5 \pm 5,8(5)$ & $<0,025$ \\
\hline Proteínas Animales & $23,0 \pm 7,0(13)$ & $29.7 \pm 4,5$ & $<0,025$ \\
\hline
\end{tabular}

$\mathrm{a}=\overline{\mathrm{X}} \pm$ D.E. (Número de Casas)

$\boldsymbol{b}=$ Significación según $t$ de Student

Tabla 8.

Resultados de laboratorio en los hijos a los 30 meses de edad

\begin{tabular}{lcccc}
\hline & Hijos adolescentes & Hijos de aduitas & P. \\
\hline Zn ug/dl & $100,7 \pm 30,0 \mathrm{a}$ & $(28)$ & $92,6 \pm 25,0(24)$ & N.S. \\
. Cu ug/d1 & $96,8 \pm 28,3$ & $(28)$ & $82,3 \pm 37,4(24)$ & N.S. \\
. Hb (g \%) & $12,6 \pm 1,4$ & $(27)$ & $12,8 \pm 9,8(23)$ & N.S. \\
Edad ósea (Meses de atraso) & $-3,4 \pm 5,8$ & $(11)$ & $-3,7 \pm 6,5(19)$ & N.S. \\
\hline
\end{tabular}

$\bar{a}=\overline{\mathrm{X}} \pm$ D.E. (Número de Casos)

Tabla 9.

Estatura promedio de los padres

\begin{tabular}{lccc}
\hline Hijos de Adolescentes & Padre & Madre & Ambos padres \\
\hline Hijos de Adultas & $\begin{array}{c}168,0 \pm 5,9(a) \\
(27)\end{array}$ & $\begin{array}{c}154,6 \pm 5,1 \\
(29)\end{array}$ & $\begin{array}{c}161,7 \pm 4,0 \\
(26)\end{array}$ \\
\hline $\mathrm{P}(\mathrm{b})$ & $\begin{array}{c}170,8 \pm 6,0 \\
(25)\end{array}$ & $\begin{array}{c}152,9 \pm 5,8 \\
(25)\end{array}$ & $\begin{array}{c}162,1 \pm 5,2 \\
(23)\end{array}$ \\
\hline
\end{tabular}

(a) $=\overline{\mathrm{X}} \pm$ D.E. (Número de Casos)

(b) = Significación según de $t S$ tudent. 


\section{DISCUSION}

Este estudio demuestra que la frenación del crecimiento que describimos anteriormente ${ }^{16}$ en los hijos de adolescentes continúa después de los 18 meses de vida, presentando un promedio estatural y un ritmo de crecimiento significativamente menor a los hijos de adultas, al continuar nuestro seguimiento longitudinal hasta los 30 meses de vida. El deterioro progresivo del NSE en la familia de las adolescentes, seria producto del mayor índice de allegamiento y de la inestabilidad laboral del jefe de familia. Es conocido, que el menor NSE aumenta la prevalencia de desnutrición, la que produce un importante retraso del crecimiento en los niños de las familias que sufren este impacto. En cste estudio de hijos de adolescentes; el déficit nutricional global especialmente calórico, pareciera no jugar un rol, puesto que no existen diferencias en la ingesta calórica y en la relación del peso para la estatura al compararlos con los hijos de adultas. Sin embargo, se encontró una significativa menor ingesta de proteínas totales $y$ de origen animal en los hijos de adolescentes que crecen con una velocidad estatural jgual o inferior al $97 \%$ de lo esperado para la edad. Los niveles plasmáticos normales de Zinc y Cobre, descartarían un déficit de minerales trazas como causa del mayor porcentaje de rétraso estatural. El no encontrar diferencias en la estatura y en el origen étnico de los apellidos de los padres de ambos grupos de niños, hace menos posible que un factor genético familiar o racial explique este retraso. También es poco posible un factor constitucional, ya que los hijos de adolescentes tienen un promedio de edad ósea similar al de los de adulta.

En resumen, hemos encontrado un menor NSE de la familia y una menor ingesta proteica del hijo de madre adolescente, factores que podrían estar jugando un rol en el deterioro del crecimiento estatural presentado por estos niños durante los 30 primeros meses de vida; al compararlos con los hijos de adultas. Es probable que este mayor retraso estatural, tenga una etiologia multifactorial y se requiera un mayor número de casos estuđiados para lograr identificarlos. Nos interesó además estudiar otros factores etiológicos como son la estimulación psicoafectiva de estos niños, cuya importancia es conocida en el normal crecimiento y desarrollo del niño ${ }^{31-34}$ Hemos estudiado las características de la relación madre--hijo en este grupo y los resultados serán publicados próximamente. El poder identificar las causas del mayor deterioro del crecimiento estatural en hijos de adolescentes permitiría implementar una adecuada prevención y/o tratamiento de este problema.

\section{RESUMEN}

Se realizó ụn seguimiento longitudinal hasta los 30 meses de vida de hijos de adolescentes de clases media-baja, para estudiar su crecimiento pondoestatural y compararlo con los hijos de adultas. Se evaluó el estado nutritivo, la estatura, el ritmo de crecimiento, la maduración ósea, los niveles plasmáticos de Zinc, Cobre y hemoglobina, la ingesta de nutrientes, la estatura de ambos padres y el rivel socioeconómico de las madres. En los hijos de adolescentes se observó menor promedio estatural, velocidad de crecimiento, ingesta proteica y nivel socioeconómico de sus madres, al compararlos con los hijos de adultas. No se encontraron diferencias en el estado nutritivo, maduración ósea, ingesta calórica, niveles plasmáticos de zinc, cobre $y$ hemoglobina $y$ en la estatura promedio de los padres. El deterioro del NSE de las madres y la menor ingesta proteica de los hijos podrian estar produciendo al menos en parte, el mayor porcentaje de retraso estatural detectado en hijos de adoles. centes al compararlos con hijos de adultas.

\section{REFERENCIAS}

1. Garn, S.M., Petzold, A.S.: Cluaracteristics of the mother and child in tecnage pregnancy. Am, J, Dis, Child. 137: $365,1983$.

2. Klein, $L_{n}$ : Early teenage pregnancy, contraception and repeat pregnancy. Am. J Obstet. Gynecol. 120: 249,1974 .

3. Dott, A.B., Fort, A.T.: Medical and social factors affecting early teenage pregnancy. Am. J. Obstet. Gy necol. 125: 532, 1976 .

4. Kaltreider, D.F., Johnson, J.W.C.: Patients at high tisk for low-birth weight delivery, Am. J. Obstet. Gynecol. 124: 251, 1976.

5. Kafotos, A.G., Christakis, G., Fordyce, M.: Nuti tion and early teenage pregnancy. En: Teenage parents and their offspring. Scort, $K, G$., Field, $T$., Robertson, E.G.: Grune and Stratton. N. York. 1981. pp. $103-127$.

6. Oppet, w.C. Royston, A.B.: Teenage birth: some social, physicological and physical sequelae. Am. J. Public Health. 61: 751, 1971.

7. Koening, M.A., Zelnik, $M_{1}$ : Repeat pregnancies among metropolitan-area teenagers: $1971-1979$. Family planning perspectives. $14: 341,1982$.

8. Osofsky, J.D., Osofsky, H.J.: Embarazo en la segunda década de la vida: consideraciones psicosociales. Clin. Obstet. Ginecol. 21: 1200, 1978.

9. Hutchins, F.L., Kendall, N., Rubino, J.: Experience with teenage pregnancy. Obstet. Gynecol. \$4: 1, 1979.

10. Statement on teenage pregnancy: Committec an Adolescence. American Academy of Pediatrics. Pediatrics 63: 795, 1979.

11. Hardy, J.B., Welcher, D. W., Staniey, J., Dollas, J.R.. Pionóstico. a largo plazo de los embarazos de adolescentes. Clin. Obstet. Ginecol. 21: 1267, 1978.

12. Thompron, R.J., Capplemon, M.W., Zeitschel, K.A.. Neonatal behavior of infants of adolescents mothers. Dev. Med Child. Neurol. 21: 474, 1979. 
13. Scholl, T.O., Decker, E.B.S., Karp, J.R., Greene, G. De Sales M.: Early adolescent pregnancy: A comparative study of pregnancy outcome in young adolescent and mature women. J. Adolesc. Health Care. 5: 00, 1984.

14. Efiong, E.I, Bajoko, M.O.: The obstetric performance of njgerian primigravidae aged 16 and under. Br. J. Obstet. Gy naecol. 82: 228, 1975.

15. Burrows, R., Muzzo, S. Liro, P., Alvarez, M.L.: Características del embarazo de la adolescente que indican riesgos de alteración en el crecimiento y desarrollo pre y post natal precoz. Informe Pro yecto $91 / 81$ DIUC, 1983.

16. Burrows, $R$, Carrasco, D., Muzzo, $S$. Crecimiento y desarrollo en hijos de adolescentes de clase media. Parte I. Rev. Chil, Pediatr. 56: 199, 1985.

17. Burrows, R.: Crecimiento Normal. En: Crecimiento y Desarrollo Normal y Patológico. Editor: $S$. Muzzo. Ediciones Instituto Profesional de Chillán. pp. $7-19,1985$.

18. Graham. G,G.: Effect of infantile malnutrition on growth. Fed. Proc. 26: 139, 1967.

19. Mönckeberg, F: La desnutrición en el niño y sus consecuencias. Rev. Cent. Estudios Educ. (México). 3: $67,1973$.

20. Craviotto, $J$.: Modificación post-natal del fenotipo causada por la Desnutrición. Gac. Med. Mex. 98: $523,1968$.

21. Sandstead, H.H., Prasad A.S, Schulert, A.R.: Hurvan zinc deficiency, endocrine manifestations and response to treatment. Am. J. Clin. Nutr. 20: $422,1967$.

22. Puentes, $R$, Uauy, $R$., Castillo, $C$.: Déficit de cobre en el lactante. Rev, Chil, Pediatr, 53: 144, 1982.

23. Hombidge, K.M. The role of zinc and other trace metals in pediatric nutrition and health. Pediatr. Clin. North. Am. 24: 95, 1977.
24. Morley, J.E., Gordon, J., Hershman, M.J.: Zinc deficiency, chronic starration and hypothalamicpituitary-thyroid function. Am. J. Clin. Nutr. 33: 1767,1980

25. Murray, R.S.: Adaptation to low-protein intakes Lancet I: $105,1969$.

26. Waterlow, J.C. Alleyne, G.A.O.: Protein malnutrition in children. Advances in knowledge in the last ten years. Medical Research Council of Great Britain. 25; $117,1971$.

27. Waterlow, J.C.: Observations on the mechanism of adaptation to low protein intakes. Lancet 23: 1091, 1968.

28. NCH-S Growth curves for children birth 18 years U.S.: Vital and Health Statistics. Serjes 11 - Number $165,1977$.

29. Alvarez, M., Wurgaft, F, Salazar, M.: Mediciones del nivel socioeconómico bajo urbano en familias con lactantes desnutridos Arch. Latinoam. Nutr. 32: 650,1982 .

30. Gretlich, W.W., Pyle, S.t.: Radiographic Allas of skeletal development of the hand and wrist. Second Edition 1959, Stanford University Press.

31. Patton, R.G., Garaner, L.I.: Deprivation dwarfism. Disordered family environment as a cause of so called idiopathic hypopituitarism. Clin. Endocrinol. 2: $132,1968$.

32. Riley, R.L., Ladwirth, J, Kaplan, S, Collip, P.F.: Failure to thrive. An Analysis of 83 cases. Californja. Med, 108: 32, 1968.

33. Rosenn, D., Stein, L., Bates, M.: Differentiation of organic form monorganic failure to thrive syndrome in infancy. Pediatrics 66: 698, 1980.

34. Mitchel, W.G., Gonell, R.W., Greenberg. R.A.: Failure to thrive: A study in a primary care setting. Epidemiology and follow up. Pediatrics 65: 971, 1980. 